\title{
A MICRO-COMPUTERIZED ANAESTHETIC RECORD SYSTEM
}

\author{
A. Zissos and L. Strunin
}

\begin{abstract}
An anesthetic record system has been implemented using a micro-computer, rather than a larger computer system. The record system provides a wide range of functions, including record retrieval, random record processing, and the generation of graphical reports on the department's operating room performance. The steps taken in realizing this system are described, with emphasis on the preparatory decisions that must be made before computer programming can be undertaken. Adopting a modest straightforward design philosophy is shown to be an important pre-requisite to success.
\end{abstract}

\section{Key Words: ANAesthetic ReCORD; MiCrocomputer; Computerized} RECORD.

In the past, the effective management of hospital anaesthetic records has proved to be an elusive goal. While the drawbacks of conventional file storage have been generally acknowledged, the traditional alternative of "buying time" on a large computer system has on the whole met with a discouraging lack of success. Part of the blame for this undoubtedly lies with over-zealous anaesthetic departments, which have frequently embarked upon computerized record schemes of unrealistic proportions. However, this failure is usually more directly attributable to the inherent complexities of large computer systems.

For the anaesthetist, the confusion generated by a remote computer installation has often proved to be a crippling burden. Typically, lacking a reasonable understanding of how the system works, one is left in an exceedingly vulnerable position. It is very easy, for example, for grievous technical misunderstandings to arise between the doctor and his programmer. Moreover, with several different groups usually sharing the same computer resources (and one's own group always seeming to have the lowest priority!), individual departments have inevitably lacked the necessary control over when and how the computer functioned. Thus, record management has often been inhibited by other totally unrelated applications.

Fortunately, recent advances in the electron-

A. Zissos, Computer Science Student, University of Calgary; L. Strunin, M.D., F.F.A.R.C.S., F.R.C.P.(C), Professor and Director, Department of Anaesthesia, University of Calgary and Foothills Hospital, Calgary, Alberta, T2N 2T9. ics industry have provided an alternative to the large computer system. The advent of the microcomputer, the availability of reliable data storage devices, and the dramatic drop in the cost of computer hardware have made it possible for individual departments to acquire their own small computer systems. These can then be dedicated to the task of record management, providing a far more workable arrangement.

Clearly, such an approach has important advantages. The micro-computer is much simpler than its large-scale predecessor, and can be mastered with relative ease. Also, the owners retain complete control over the actual computer hardware, and so have little difficulty gaining access to it, or specifying its operation.

Just such a micro-computer-based anaesthetic record scheme has been implemented by the Department of Anaesthesia at Foothills Hospital, Calgary. The aim was to provide graphical summaries of the department's operating room performance for any chosen period of time. In addition, easy access was needed to individual anaesthetic records, as was the ability to retrieve groups of records through the specification of a set of common characteristics. In short, an attempt was made to provide the department with the type of record processing functions that have traditionally, and perhaps inappropriately, been assigned to the large centralized hospital computer.

\section{METHODS}

The first matter to be settled was which

Can. Anaesth. Soc. J., vol. 29, no. 2, March 1982 


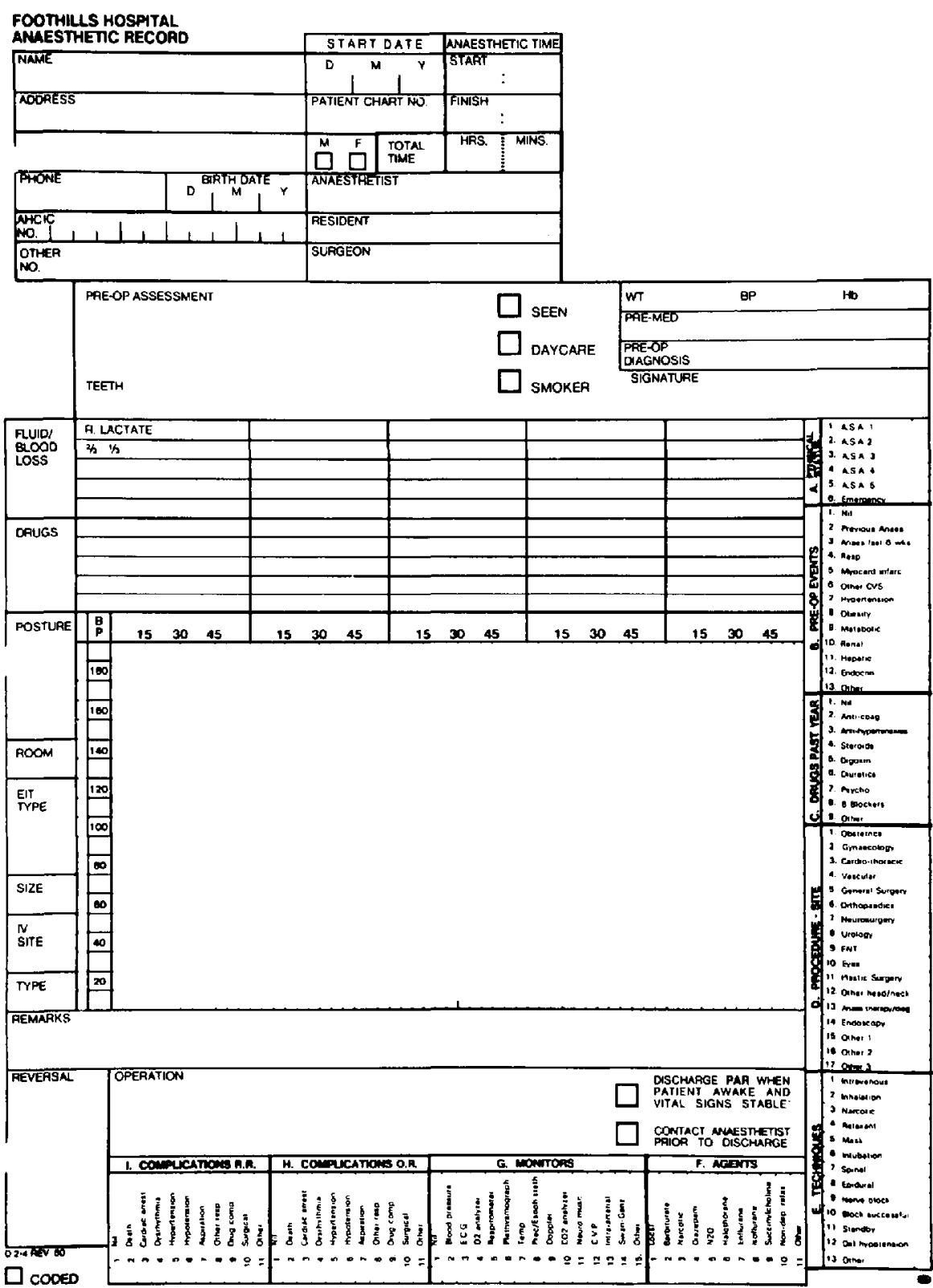

FIGURE 1 The anaesthetic record in current use at Foothills Hospital. This record includes the information required by the computer and a conventional patient management chart. The sheet is a no-carbon type; the original remains in the patient record and the copy is used to enter data into the computer. 


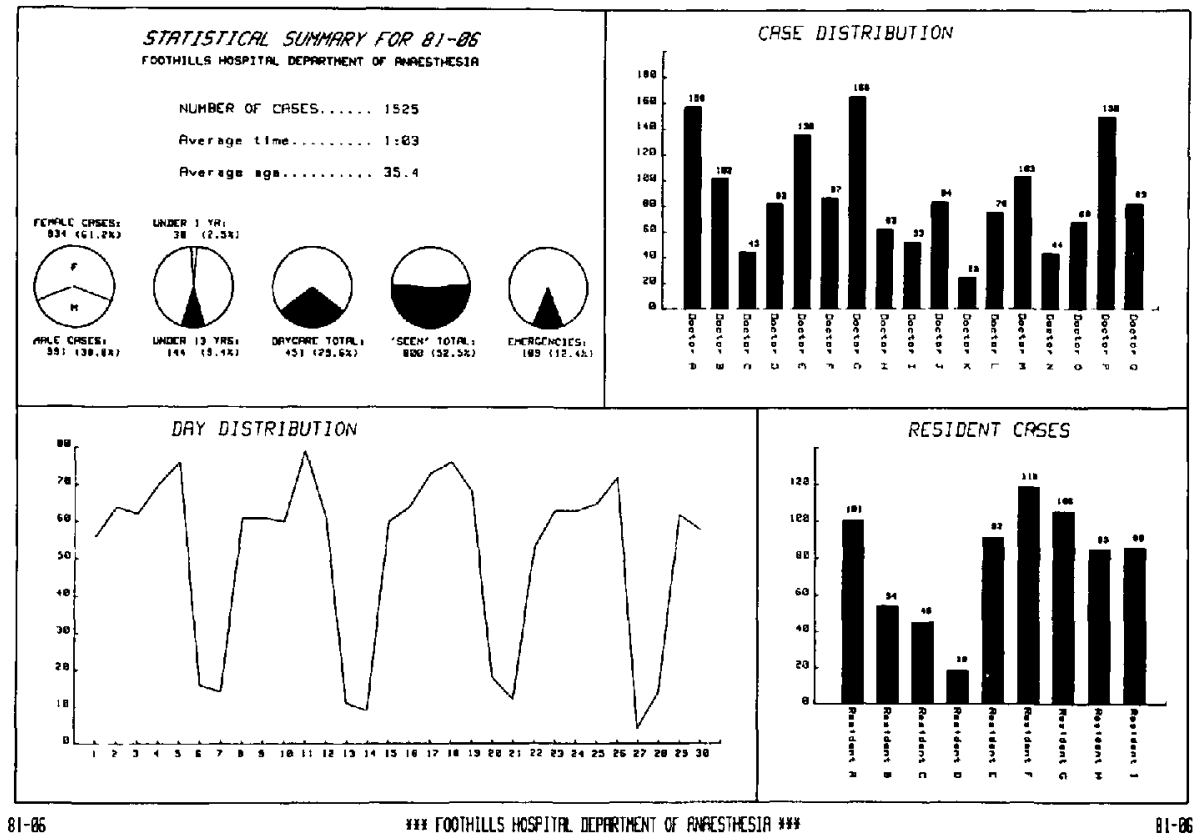

Figure 2a

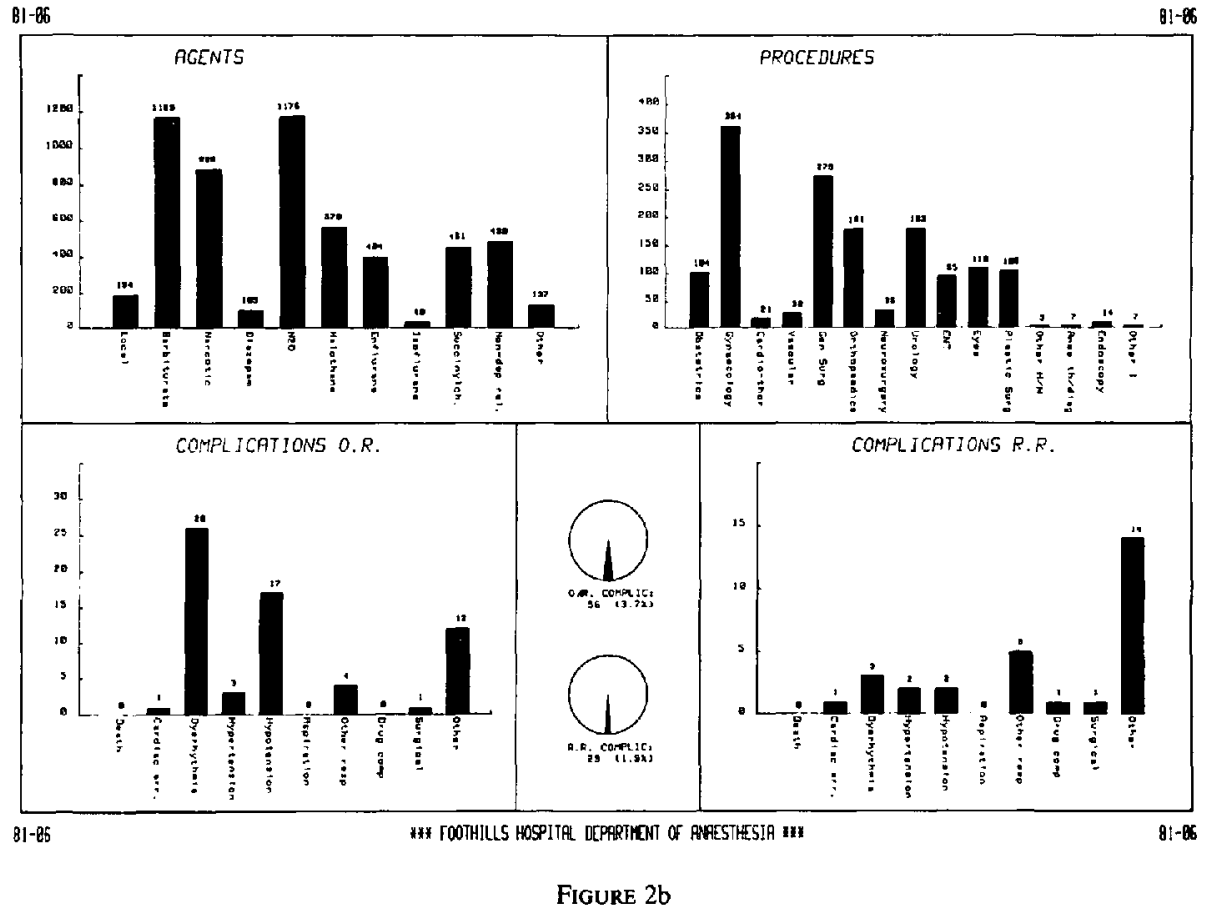


91-x

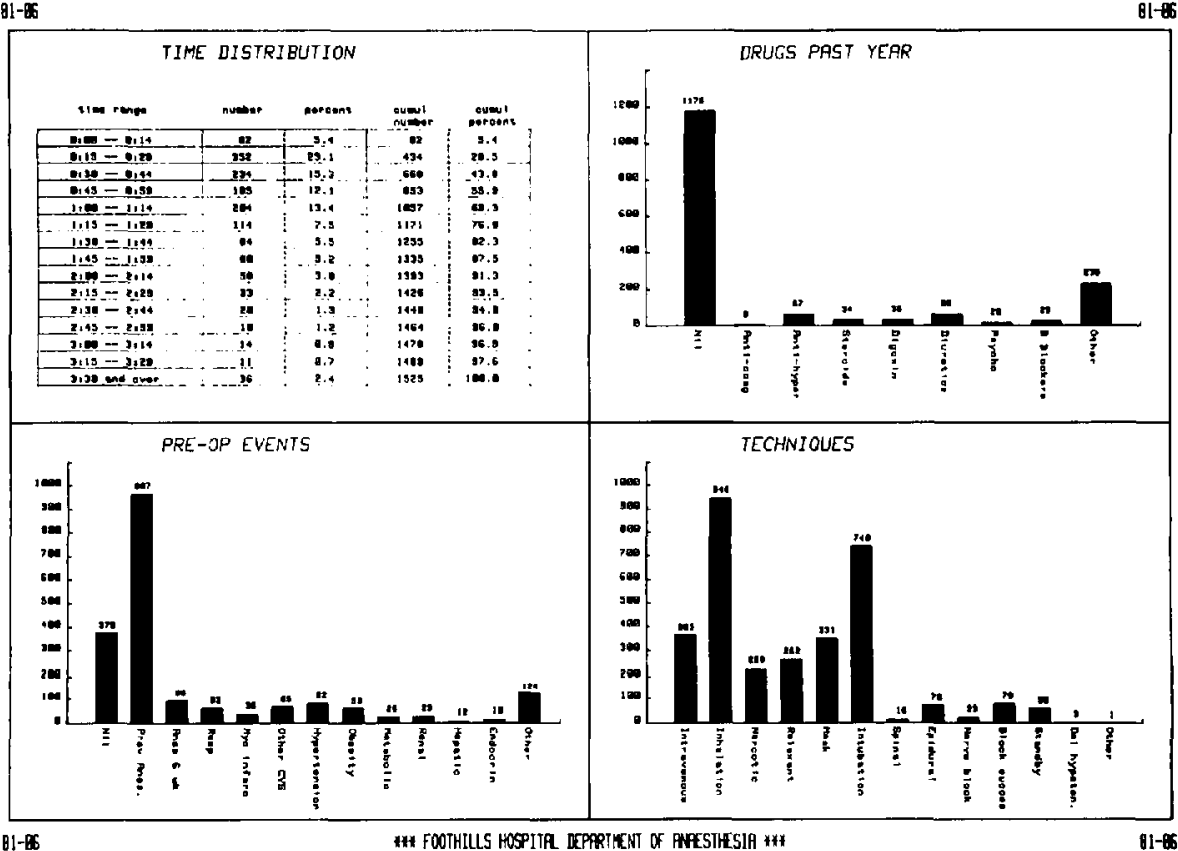

FIGURE 2c

FIGURE $2(a, b, c)$ These show the graphical report generated by the computer system for the month of June, 1981.

micro-computer system to use. In making this decision the department was influenced by Dr. John Paull of the Royal Women's Hospital in Melbourne, who has recently implemented an anaesthetic billing system on a Hewlett-Packard desktop computer (H.P. series 9845B). ${ }^{1}$ The same machine was ultimately chosen, not only on the strength of this precedent, but also because it exhibited a unique combination of desirable characteristics.

The HP 9845B is exceedingly easy to operate. More importantly, it has an excellent selection of peripheral devices. Of these the project uses a large disk drive (20 megabytes) for record storage, a printer and a colour plotter for report generation. Moreover, these peripherals are easily transferable to a number of other HewlettPackard computers, an important consideration for eventual upgrading of the system.

Weighed against these positive considerations were certain drawbacks which are in fact typical of today's micro-computers. The HP 9845B cannot support multiple terminals, so that only one person can use the record system at any given time. Although this was of no immediate concern, it had to be borne in mind when considering future expansion. A more serious drawback is the relatively slow processing speed (for example, generating an extensive report on 1000 records requires 12 minutes), which places practical limitations on just how much record manipulation can be done without unduly tying down the system. However, this is also of little consequence for a single department, as the computer is dedicated to the task of record management alone. Finally, it should be appreciated that, because the department is actually housing the entire computer system, the stored data is somewhat less secure than it would be on a more remote installation. The only way to secure this type of computer fully would be to restrict physical access.

Having settled upon a computer system, it was now time to determine exactly what information it was to process. In making this decision it was most important, for two reasons, that a spirit of compromise should prevail. First, certain types of data are particularly suitable for computerized record handling, whereas others may be considerably less so. In particular, information of a 
Boolean (yes-or-no) nature can be stored and manipulated very efficiently, as can most data with definite limits to size or value (such as chart numbers and dates, etc.). On the other hand, graphical data and data without easily defined limits (such as verbal descriptions) tend to be far more difficult to accommodate. So it was necessary to some extent to sacrifice non-essential data that presented storage or computational problems, and thereby favour data of a more convenient type. Second, and more importantly, the copying of anaesthetic record data into the computer represented an additional burden for the department's secretarial staff, and so the data had to be both quick and easy to enter. Thus lengthy entries such as names and addresses were ruled out.

As the current anaesthetic record had very little suitable information on it, it became obvious that a new and more appropriate form would have to be designed. Two proposals were considered: a relatively simple form based on the one in use at the Health Sciences Centre in Winnipeg ${ }^{2}$ and a considerably more complex form described by Cook and Herter. ${ }^{3}$ Both were suitable in so far as they incorporated a large amount of Boolean information, the difference between them being that the former categorized cases in a highly general manner, whereas the latter took a far more exhaustive approach. While the more complex form obviously held far more useful information, it was quickly rejected on the grounds of tedium. Given the case load of the present anaesthetic staff it did not seem reasonable to burden them with so lengthy a form. In addition, the integrity of record data would have proved most difficult to maintain, as increasing the record's detail undoubtedly increases propensity to error in filling it out.

So, having settled on a new anaesthetic record, it was time to return to the problem of what information was to be stored in the computer system. In effect this was the final weeding out process; a stripping down of the form to arrive at its essential (and expedient) descriptive components.

In line with the department's limited objectives, the only patient identification information included was the hospital number, birthdate, and sex. The rest of the computer record, a description of the anaesthetic procedure, was limited to the case blocks (A-I; see Figure 1), two additional Booleans (seen?; daycare?), the anaesthetist and resident identification numbers, the operating room number, the date, and finally the duration of the anaesthetic.

Once running, it was determined that about two hours of operator time are needed daily to handle a typical batch of seventy anaesthetic records. This includes both entering the records into the computer, and then carrying out "backup" operations to guard against possible data loss. With such a low entry overhead there is ample time in the working day for others to use the system.

\section{RESULTS}

As a result of the decision to limit the anaesthetic record, the information provided by the computer system is of a rather general nature, making it impossible to access groups of records by highly precise specifications. This was a necessary concession to simplicity and realism; making such decisions at the beginning ensured the trouble-free development of a fully workable system with a minimum amount of additional effort from department members.

Another important result of the simplistic approach was to render the record system as a whole remarkably streamlined; in no sense, for example, is there any redundant information in the computer record. Moreover, every data item serves a specific purpose, either in single or grouped record retrieval, and all but two (hospital and room numbers) figure in the periodic graphical reports of the department's performance (Figure 2).

The graphical reports are the comerstone of the system's application. To begin with, they provide useful administrative information, such as the numbers of each sex treated, the percentages of daycare, seen and emergency patients, the average anaesthetic time and a time breakdown, as well as daily, monthly or yearly case totals. In addition, they provide the anaesthetists and residents with feedback on their own work, including breakdowns of the types of procedures and monitors used. This is particularly useful for residents, who can then use the system to retrieve the records of all cases in which they were involved and, thus, easily chart their own progress.

Finally, the graphical reports provide patient data and information concerning complication rates which facilitates the task of quality control. On a quarterly basis the computer is made to generate a graphical report for the previous three 
months, which is then used for the departmental morbidity and mortality rounds. If necessary, statistical breakdowns can be provided for any group of records under study. For a more detailed study, the computer can print out its own records, or provide identifying information so that the original records can be retrieved readily.

\section{Discussion}

The actual implementation of the project, from conception to completion, took about eight months. Of this period, roughly a quarter was devoted to logistical considerations such as acquisition of the computer hardware and the selection of the new anaesthetic form. The computer programs were written by one of the authors (AZ), and required five months of full-time work.

An important consideration here was that the system be implemented as three essentially independent modules; one for entering, changing, and finding records (record management), the second for processing groups of records, and the third for generating the graphical reports. Thus, by implementing the record management module first, it was possible to start entering trial records into the computer well before the entire system had been completed.

At the present time the system has been running for almost a full year. The only difficulties experienced so far have involved a certain degree of inconsistency on the part of the anaesthetists in filling out one or two sections of the anaesthetic form. The fault here lies with the wording of the anaesthetic record, and revisions are being made.

As for the future, a gradual enhancement of the system is forseen, using the experience gained through this pilot project. To cope with projected new applications it will, as expected, become necessary to upgrade the processor (but not, as mentioned earlier, the peripheral devices) from a micro- to a mini-computer. With more people likely to be using the system, multiple terminals and rapid processing speed will become essential.

The immediate aim is to include linkage to another hospital and government billing among the computer's duties. These and other additions will inevitably rob the system of its present commendable simplicity. But by making such enhancements in a slow, methodical fashion, and by always building on the existing base, it seems likely that the pitfalls associated with most complex computing schemes will be avoided.

\section{REFERENCES}

1. Paull, J.D. A computerised anesthetic record and billing system. Excerpta Medica, International Congress Series 533: 535 (1980).

2. Burnham, M. \& Craig, D.B. A postanaesthetic follow-up program. Can. Anaesth. Soc. J. 27: 164-168 (1980)

3. COOK, G.B. \& HERTER, V.L. An improved anesthesia record. Anesthesiology 32: 367-368 (1970).

\section{RÉsumé}

On a utilisé un micro-ordinateur plutôt qu'un ordinateur de grande capacité pour la collecte et compilation des données anesthésiques incluant la récupération et le traitement des données et la réalisation de rapports graphiques sur la performance du département en salle d'opération. Les auteurs décrivent les étapes de réalisation du système en insistant sur les décisions qui doivent être prises avant de commencer la programmation. Le succès d'une telle entreprise nécessite une approche directe avec des objectifs modestes. 